\title{
The CASPA Tool: Causality-Based Abstraction for Security Protocol Analysis
} Tool Paper

\author{
Michael Backes ${ }^{1,2}$, Stefan Lorenz ${ }^{1}$, Matteo Maffei $^{1}$, and Kim Pecina ${ }^{1}$ \\ ${ }^{1}$ Saarland University, Saarbrücken, Germany \\ ${ }^{2}$ MPI-SWS
}

\begin{abstract}
CASPA constitutes a push-button tool for automatically proving secrecy and authenticity properties of cryptographic protocols. The tool is grounded on a novel technique for causality-based abstraction of protocol executions that allows establishing proofs of security for an unbounded number of concurrent protocol executions in an automated manner. We demonstrate the expressiveness and efficiency of the tool by drawing a comparison with T4ASP, the static analyzer for secrecy properties offered by the AVISPA tool. CASPA is capable of coping with a substantially larger set of protocols, and excels in performance.
\end{abstract}

\section{Introduction}

Proofs of security protocols are known to be error-prone and, owing to the distributed-system aspects of multiple interleaved protocol runs, awkward to do. In fact, vulnerabilities have accompanied the design of such protocols ever since early authentication protocols like the Needham-Schroeder protocol, to carefully designed de-facto standards like SSL and PKCS, up to current widely deployed products like Microsoft Passport. Formal methods have proved to be salient tools for dealing with such flaws, by helping both to securely design and to analyze security protocols, and even to formally establish their security properties.

A central intricacy that these tools have to tackle is to concisely treat the potentially very large number of concurrent protocol executions. We have developed CASPA (Causality-based Abstraction for Security Protocol Analysis), a tool for establishing formal security proofs of cryptographic protocols for an unbounded number of concurrent protocol executions in a mechanized manner. The tool is grounded on a recently proposed abstract interpretation of cryptographic protocols 3] based on causal graphs. Causal graphs are finite dependency graphs in which nodes represent process events and edges express the causality among events. These graphs constitute a sound abstraction of an unbounded number of protocol executions and, interestingly, they serve as a graphical illustration of the actual protocol behavior. A quick inspection of these graphs often suffices to identify unintended, and possibly harmful, interactions among parties. This facilitates protocol design and error detection even on the human level.

A. Gupta and S. Malik (Eds.): CAV 2008, LNCS 5123, pp. 419 422, 2008.

(C) Springer-Verlag Berlin Heidelberg 2008 
Related Work. We demonstrate the expressiveness and efficiency of our tool by drawing a comparison with T4ASP [1319], the static analyzer for secrecy properties offered by AVISPA [2, the well-known tool suite for security protocol analysis. CASPA is capable of coping with a substantially larger set of protocols than TA4SP, and it furthermore excels in terms of performance; moreover, CASPA is capable of verifying both secrecy and authenticity properties in contrast to only secrecy properties in the case of TA4SP. Note that TA4SP so far constitutes the only tool of the AVISPA tool suite that is capable of producing proofs of security; the remaining tools such as OFMC [5] rely on state-space exploration techniques and are constraint to either taking into account only a limited number of concurrent protocol executions, typically two or three, or giving up guaranteed termination in general. The same holds for other techniques based on state-space exploration such as Athena [16, CPSA [12], and Scyther [11. ProVerif [6] is a tool based on Horn-clause resolution that verifies trace-based security properties, such as secrecy and authenticity, as well as observational equivalence of process behavior, is very efficient, but provides guaranteed termination only for tagged protocols [7]. The Lysa tool [8] implements a control-flow analysis that offers termination and security proofs for an unbounded number of protocol executions, but does not support security properties based on correspondence assertions [17.

\section{The CASPA Tool}

CASPA is written in Objective CAML and equipped with a graphical user interface, assisting the user in the specification and in the analysis of cryptographic protocols, see Figure 1. CASPA provides an editor for protocol specifications, offering a quick loading procedure for the protocols specified in underlying protocol libraries, and a convenient parsing procedure for user-defined protocol specifications. In addition, the tool

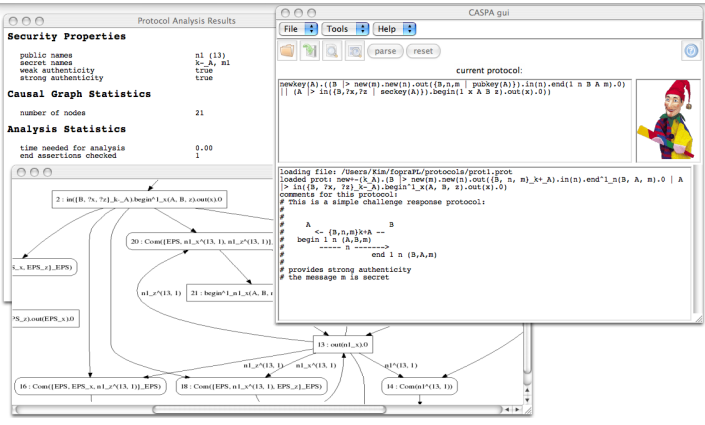

Fig. 1. The CASPA Tool features a graph management system that automatically generates and displays causal graphs. Finally, CASPA offers a fully mechanized analyzer that verifies secrecy and authenticity properties on a given causal graph and displays the results. More precisely, CASPA allows for analyzing the security properties secrecy, weak authenticity, and strong authenticity [14: as in AVISPA, authenticity properties are specified in terms of correspondence assertions [17]. Since causal graphs are of finite size, the analysis is assured to terminate. The analysis entails security proofs that establish the safety of the protocol for a potentially unbounded 
Table 1. Protocol results, conducted on a Pentium-IV 3GHz 1GB under linux

\begin{tabular}{||l|c|c|c|l||c|c|c||}
\hline Protocol & CASPA & TA4SP & OFMC & Protocol & CASPA & TA4SP & OFMC \\
\hline CHAPv2 & $0,93 \mathrm{~s}$ & $10,59 \mathrm{~s}$ & $0,32 \mathrm{~s}$ & NSPK & $0,13 \mathrm{~s}$ & $7,56 \mathrm{~s}$ & $0,01 \mathrm{~s}$ \\
CRAM-MD5 & $0,09 \mathrm{~s}$ & - & $0,71 \mathrm{~s}$ & NSPK-KS & $28 \mathrm{~m}$ & - & $1,1 \mathrm{~s}$ \\
EKE & $0,81 \mathrm{~s}$ & $7,56 \mathrm{~s}$ & $0,19 \mathrm{~s}$ & NSPK-fix & $0,08 \mathrm{~s}$ & $0,98 \mathrm{~s}$ & $0,18 \mathrm{~s}$ \\
IKEv2-CHILD & $0,31 \mathrm{~s}$ & - & $1,19 \mathrm{~s}$ & NSPK-KS-fix & $7 \mathrm{~m}$ & - & $24,86 \mathrm{~s}$ \\
ISO1 & $0,05 \mathrm{~s}$ & $\times$ & $0,02 \mathrm{~s}$ & SHARE & $0,4 \mathrm{~s}$ & $14,38 \mathrm{~s}$ & $0,08 \mathrm{~s}$ \\
ISO3 & $1,08 \mathrm{~s}$ & $\times$ & $0,04 \mathrm{~s}$ & UMTS-AKA & $0,04 \mathrm{~s}$ & $0,51 \mathrm{~s}$ & $0,02 \mathrm{~s}$ \\
LPD-MSR & $0,05 \mathrm{~s}$ & - & $0,02 \mathrm{~s}$ & APOP & $0,44 \mathrm{~s}$ & $\times$ & $2,94 \mathrm{~s}$ \\
LPD-IMSR & $0,37 \mathrm{~s}$ & - & $0,08 \mathrm{~s}$ & DHCP-DA & $1,03 \mathrm{~s}$ & - & $0,06 \mathrm{~s}$ \\
\hline
\end{tabular}

number of protocol executions. As usual for static analysis techniques and due to the undecidability of the security problem, false positives may occur caused by an insufficient precision of the analysis, hence potentially classifying secure protocols as insecure. The CASPA tool is freely available at 4 .

\section{Performance Evaluation of CASPA}

We evaluate the performance of our tool by running it on a subset of the AVISPA library. The facilitate our experiments, we developed a translator from the Intermediate Format protocol language [2] that the AVISPA suite is based upon into the dialect of the spi-calculus [1] used in our tool. The translation is only partially automated in that it requires some manual steps, such as specifying the owners of the keys and defining suitable correspondence assertions. These steps were straightforward in all protocols considered so far, but they admittedly require basic familiarity with our language and understanding of the protocol.

The results are reported in Table 1] The tool succeeded in the analysis of safe protocols (i.e., we did not get any false positives), and it failed to establish security proofs of flawed protocols as expected. For each protocol, the table reports the running time for CASPA and TA4SP. The performance evaluation shows that CASPA is capable of dealing with a substantially larger set of protocols than TA4SP: the symbol - means that the protocol is not supported by the tool, while the symbol $\times$ means that the protocol guarantees only authenticity properties, which can be verified by CASPA and not by TA4SP. In addition, the evaluation shows that even for the protocols that are in scope of both TA4SP and CASPA, the CASPA tool improves in terms of performance. Note that the subset of protocols we consider comprises all protocols of the AVISPA library for which an analysis with TA4SP succeeds. Some protocols in the AVISPA library employ non-standard equational theories that are not supported by our tool (e.g., NSPKxor that is based on xor) and for some other protocols the analysis did not succeed due to memory exhaustion (e.g., SET and TSL).

For comparison, we additionally depict the running times of OFMC [5], the most advanced model checker in the AVISPA tool, when the analysis is constrained to three executions. In this restricted setting, OFMC shows significantly better performances. Scaling this approach to more executions rapidly becomes infeasible, hence leaving potential attacks undetected (e.g., cf. [10 15]). 


\section{References}

1. Abadi, M., Gordon, A.D.: A calculus for cryptographic protocols: The spi calculus. Information and Computation 148(1), 1-70 (1999)

2. Armando, A., Basin, D., Boichut, Y., Chevalier, Y., Compagna, L., Cuellar, L., Drielsma, P., Heám, P., Kouchnarenko, O., Mödersheim, J.M.S., von Oheimb, D., Rusinowitch, M., Santiago, J., Turuani, M., Viganò, L., Vigneron, L.: The avispa tool for the automated validation of internet security protocols and applications. In: Etessami, K., Rajamani, S.K. (eds.) CAV 2005. LNCS, vol. 3576, pp. 281-285. Springer, Heidelberg (2005)

3. Backes, M., Cortesi, A., Maffei, M.: Causality-based abstraction of multiplicity in cryptographic protocols. In: Proc. 20th IEEE Symposium on Computer Security Foundations (CSF), pp. 355-369. IEEE, Los Alamitos (2007)

4. Backes, M., Lorenz, S., Maffei, M., Pecina, K.: The CASPA tool, www.infsec.cs.uni-sb.de/caspa

5. Basin, D.A., Mödersheim, S., Viganò, L.: Ofmc: A symbolic model checker for security protocols. IJIS 4(3), 181-208 (2005)

6. Blanchet, B.: An efficient cryptographic protocol verifier based on Prolog rules. In: Proc. 14th IEEE Computer Security Foundations Workshop (CSFW), pp. 82-96. IEEE, Los Alamitos (2001)

7. Blanchet, B., Podelski, A.: Verification of cryptographic protocols: Tagging enforces termination. In: Gordon, A.D. (ed.) ETAPS 2003 and FOSSACS 2003. LNCS, vol. 2620, pp. 136-152. Springer, Heidelberg (2003)

8. Bodei, C., Buchholtz, M., Degano, P., Nielson, F., Nielson, H.R.: Static validation of security protocols. Journal of Computer Security 13(3), 347-390 (2005)

9. Boichut, Y., Genet, T.: Feasible trace reconstruction for rewriting approximations. In: Pfenning, F. (ed.) RTA 2006. LNCS, vol. 4098, pp. 123-135. Springer, Heidelberg (2006)

10. Chadha, R., Kremer, S., Scedrov, A.: Formal analysis of multi-party contract signing. In: Proc. 17th IEEE Computer Security Foundations Workshop (CSFW), pp. 266-279. IEEE, Los Alamitos (2004)

11. Cremers, C.: Scyther - Semantics and Verification of Security Protocols. Ph.D. dissertation, Eindhoven University of Technology (2006)

12. Doghmi, S., Guttman, J., Thayer, F.: Searching for shapes in cryptographic protocols. In: Grumberg, O., Huth, M. (eds.) TACAS 2007. LNCS, vol. 4424, pp. 523-537. Springer, Heidelberg (2007)

13. Genet, T., Tong, V.: Reachability Analysis of Term Rewriting Systems with Timbuk. In: Nieuwenhuis, R., Voronkov, A. (eds.) LPAR 2001. LNCS (LNAI), vol. 2250, pp. 695-706. Springer, Heidelberg (2001)

14. Lowe, G.: A Hierarchy of Authentication Specification. In: Proc. 10th IEEE Computer Security Foundations Workshop (CSFW), pp. 31-44. IEEE, Los Alamitos (1997)

15. Millen, J.: A necessarily parallel attack. In: Proc. of Workshop on Formal Methods and Security Protocols (1999)

16. Song, D.X.: Athena: a new efficient automatic checker for security protocol analysis. In: Proc. 12th IEEE Computer Security Foundations Workshop (CSFW), pp. 192 202. IEEE, Los Alamitos (1999)

17. Woo, T.Y.C., Lam, S.S.: A lesson on authentication protocol design. Operation Systems Review 28(3), 24-37 (1994) 